\title{
Mode of Birth and Infant Outcomes: A Review of Literature
}

\author{
Amanda Burke-Aaronson* \\ Assistant Professor in the School of Nursing and Health Professions at University of San Francisco
}

Received: November 2, 2016; Accepted: January 22, 2017; Published: March 24, 2017

*Corresponding author: Amanda Burke-Aaronson, Assistant Professor, School of Nursing and Health Professions University of San Francisco, 2130 Fulton St, San Francisco, CA 94117-1080;E-mail: acaaronson@usfca.edu

\begin{abstract}
Trauma in birth can be stressful for the newborn. With consideration to the theory of allostatic load, this critical review of literature addresses the potential for a lasting effect on infant temperament, breastfeeding and sleep based on mode of birth. I used CINAHL, Pubmed and PsycInfo databases, and the keywords "birth, outcomes, adaptation, lactation, breastfeeding, infant, sleep, temperament", initially with no limitations on dates. However, ultimately a limitation on dates for "breastfeeding" was applied because of cultural shifts about infant feeding. All articles found addressing mode of birth and the listed outcomes were included. Further research is needed in all of these areas, as the current body of literature is small and inconclusive. However, there is some suggestion from the evidence of an affect of mode of birth on breastfeeding, and infant sleep. This would indicate that decreasing trauma in birth is best for newborn adaptation.
\end{abstract}

Keywords: Mode of birth; Newborn adaptation; Newborn stress; Parturition

\section{Background}

Mode of birth (MOB), differentiated as either spontaneous vaginal, instrument vaginal, or cesarean (emergency or planned), has the potential to have lasting repercussions for the infant, as well as the mother - which then can also affect the newborn. A woman's postpartum lack of mobility (as in the case of cesarean birth, or epidural usage) and/or pain can affect her ability to care for her infant, which in turn may risk not meeting the physical (specifically, nutrition and hygiene) or emotional needs of her baby. For the neonate, normal adaptation includes respiratory, circulatory, thermoregulatory and metabolic changes. In an otherwise healthy pregnancy, the addition of labor interventions can increase the risk of a difficult transition to extra uterine life for the newborn [1, 2]. Cesarean births are now approximately $32 \%$ of all births in the United States [3]. This rise does not appear to be related to an increase in pregnancy risk factors [4].

Cesarean birth can result in decreased fluid clearance from the newborn's lungs, increasing risk of transient respiratory problems. Hypothermia is a risk in a cold operating room. Cesarean birth typically results in separation between the mother and infant, which can delay or inhibit the onset of breastfeeding. Without early colostrum, glucose stores are depleted. Signs of stress exhibited by the newborn can include poor muscle tone, low heart rate, and poor spontaneous respiration, or increased work of breathing $[5,6]$.

Vacuum extraction can result in cephalohematoma, subgaleal bleeds, or shoulder dystocia (and subsequently clavicular fracture). Forceps carry the risk of facial nerve palsy (approximately 1\% of all forceps births), bruising, and intracranial bleeding [7].

The purpose of this critical review of the literature was to explore the existing literature on MOB and neonatal outcomes, and identify gaps in knowledge for future research. The question to be answered is: "in the newborn population, does mode of birth (spontaneous vaginal, instrument vaginal or cesarean) have a lasting effect on infant temperament, breastfeeding and sleep?"The theory of allostasis [8] was used to organize this review of literature.

\section{Theoretical Approach to the Literature Review}

Labor is a natural and necessary stress that alters vital functions for transition to extra uterine life. Boldt and colleagues [9] discovered that normal labor and birth resulted in elevated infant cord blood levels of adrenomedullin, a potent pulmonary vasodilator, when compared to cesarean births without labor. This supports the physiologic role that normal labor stress plays in neonatal adaptation. However, additional birth trauma may have lasting repercussions [8]. A review by Anand and Scalzo [10] describes such lasting repercussions as altered responses to pain, behavioral and emotional problems in childhood, major psychoses, anxiety/depression, and suicide in adolescence and adulthood.

In the theory of allostatic load, stress is best described as "the strain on the body produced by repeated ups and downs of physiologic response, as well as by the elevated activity of physiologic systems under challenge, and the changes in metabolism and the impact of wear and tear on a number of organs and tissues, (which can) predispose the organism to disease" (page 2094) [11]. Physical stress during childbirth is part of the natural birth process and it is assumed that a spontaneous vaginal birth places the least amount of strain on the fetus. High allostatic load from surgical intervention may result in many adverse health outcomes. This theory emphasizes the importance of minimizing sources of undue stress on the body. 


\section{Literature Review}

Using CINAHL, PubMed, and PsycInfo databases, I entered the keywords "birth, outcomes, depression, adaptation, lactation, breastfeeding, infant, sleep, temperament" with no limitations on dates, though limited to the English language. Ultimately, "breastfeeding" included a limitation on dates because of cultural shifts about methods of infant feeding. All studies found pertaining to mode of birth and the outcome variables listed were included, again with the date limitation for breastfeeding. This search was last run in June 2015. Data from each article were organized in a table for comparison. Bias risk was included in the "study limitations" portion of the grid, and is listed for each article. The majority of articles used categorical variables, and many were dichotomous. Due to ethical considerations, no randomized, controlled studies can be done with this vulnerable population. There is no conflict of interest in this review.

\section{Mode of birth and new born temperament}

Newborn temperament has been infrequently studied with regard to MOB. The following two (older) studies evaluated infant temperament as it relates to the birth experience.

In a randomized clinical trial, women were assigned to either Leboyer technique births or to "gentle but conventional" births [12]. Outcomes of interest included neonatal irritability and responsiveness, maternal experience and perception of her birth, and her perception of her infant. Prior to randomization in the $3^{\text {rd }}$ trimester, the mothers completed a questionnaire about health status and attitudes toward pregnancy and birth. At 36 weeks, mothers were stratified by parity and social class and then randomized to the Leboyer technique or "gentle" control. Birth conditions were well controlled. In the Leboyer births, the room was kept at $27^{\circ} \mathrm{C}$, and was dimly lit with one lamp. Labor and birth occurred in the same bed, with a single sterile sheet placed under the mother's buttocks. The baby was placed skin-to-skin on mom's abdomen and massaged by the mother immediately after birth. The umbilical cord was cut when it stopped pulsing, and the father bathed the newborn in warm water. Sound levels were kept minimal. Conventional births took place in a birth room kept at $24^{\circ} \mathrm{C}$, lit by overhead fluorescent lights, with full sterile drapes, the cord was cut within 60 seconds, and the baby was wrapped in a blanket and returned to the mother. No bath was given, and no limitation was placed on sound levels. Bulb suctioning was done as necessary and weighing and silver nitrate eye drops were deferred until after the first hour in both groups. All obstetricians scrubbed and gowned in the same manner. All infants were handled gently and infant/parent interaction was facilitated. Records were kept of membrane status, anesthetic/ analgesic use, and additional neonatal treatments. If forceps or cesarean birth occurred or if the baby could not remain with the mother, the protocol was not completed.

These authors indicated that 20 infants per group would be needed to detect a large effect size for statistical significance. Due to attrition or missing data, there were not always 20 infants per group for all observations. Additionally, both groups consisted of mothers requesting the Leboyer technique, and therefore care once the dyad was home may have affected long term outcomes. There were no were significant differences between the two groups, including infant temperament, and the small sample may have contributed to a type 2 statistical error, in which there may be a difference that is too small to detect in this sample.

In a follow up study, another group of researchers in Quebec looked at infant temperament in relation to gentle birth techniques (non-standardized, though clearly defined by the authors) and other parental risk factors [13]. They had a convenience sample of 49 infants in the gentle birth group and 263 in the non-gentle birth group. Using the well-validated Infant Temperament Questionnaire, they rated infants as either "easy" or "difficult" and compared them on birth techniques, gender, rooming-in or not, and MOB. Even in this large sample, findings support the previous study [12], indicating that the gentle and non-gentle groups did not differ on temperament for infants at four or eight months of age.

\section{Mode of birth and breastfeeding}

Due to changing attitudes about breastfeeding, both in society and in the hospital, studies evaluated in this review pertaining to breastfeeding and MOB are limited to publications since 1999, and to studies performed in the United States. However, one study published in 1969, which evaluated the physiologic basics of newborn sucking, and one study pertaining to the physiology of lacto genesis, are included.

The 1969 study combined data from two studies and divided 210 infants by birth risk factors into a "suspect" group compared to a "normal" birth group [14]. Risk factors in the "suspect" group included antepartum hemorrhage, <37 weeks gestation, >43 weeks gestation, $>2$ hours second-stage labor, mid forceps birth, breech birth, posterior presentation, precipitate birth, cesarean birth, "birth injury" (not otherwise specified), birth weight less than 2,500 grams, one minute Apgar score $<7$, "abnormal signs" in the baby (not otherwise specified), and initial treatment in the intensive care unit. All babies were evaluated before their first feeding in the laboratory for "dry" (non-nutritive) sucking on a nipple that registered pressure exerted and sucking time (time spent sucking during the last 90 seconds of a two minute trial). After this baseline was established, 94 babies were given an additional two minutes sucking during which 0.5 milliliters of $5 \%$ dextrose water was delivered every tenth suck. Shorter sucking time was related to short labor duration, spontaneous birth, low forceps, and maternal anesthetic use. Reduced caloric and volume intake was also related to short labors, as well as maternal anesthetic use. All parental factors except for type of birth and sex were significant for feeding behaviors when formula feeding. Sucking behaviors were significantly related to labor duration, type of birth and anesthesia. Non-nutritive sucking was not significantly influenced by labor risk factors. Major limitation to this study was the pre-feeding intervention. Artificial nipples are currently discouraged in breastfeeding newborns, as the physiology of sucking on an artificial nipple is different from suckling on a breast. Therefore, it is possible that the use of an artificial nipple may not reflect breastfeeding-sucking physiology, and further may affect subsequent breastfeeding in some infants. 
The second early study (1999) was a prospective longitudinal study in which 192 women were recruited in Connecticut from 1996-1997 to evaluate risk factors for delayed onset of lactation (DOL) [15]. DOL was defined as greater than 72 hours, and onset of lactation ("milk coming in") was by maternal report. Exclusive formula-feeding prior to onset of lactation, infant less than $8 \mathrm{lb}$ at birth, unscheduled cesarean birth (but not elective cesarean birth), prolonged stage 2 labor, maternal obesity, and white or Hispanic ethnicity were risk factors for DOL. Women in the study were nulli- and multi-parous, mostly Caucasian, moderately well educated, and the majority had anesthesia in birth. While not all intended to breastfeed, over $75 \%$ were breastfeeding on day two. Medical complications were not a risk factor for DOL. Authors intentionally included a higher proportion of women who were nulliparous and who underwent cesarean birth in their sample. The authors attributed DOL to emergency cesarean birth and prolonged stage 2 labor. They did not address pre-labor feeding plans.

In a study of 280 women in a more rural area of California, cesarean birth was also found to be an obstacle to early breastfeeding success, along with nulliparity, duration of labor, labor medications, and formula use [16]. Outcomes of interest were "Suboptimal Infant Breastfeeding Behavior" (SIBB), DOL, and excess infant weight loss on day three (defined as a loss of $10 \%$ or more since birth). Investigators found that cesarean birth was a significant risk factor for SIBB in the first 24 hours of life, and DOL. SIBB was defined as an Infant Breast Feeding Assessment Tool (IBFAT) score ten or less. A significant interaction was found for parity and MOB. Infants born vaginally to multiparous mothers were at the lowest risk for SIBB. Infants born vaginally to primiparas had 1.72 times the risk of SIBB (95\% CI, 1.28-2.09, $\mathrm{p}=.001)$, and those born by cesarean to multiparas were at 2.46 times the risk of SIBB (95\% CI, 1.31-2.74, $\mathrm{p}=.02$ ).

One large $(n=3026)$ longitudinal study (Infant Feeding Practices II) looked at MOB and breastfeeding outcomes, by selfreport, at four weeks and six months [17]. Women also reported if they intended to breastfeed (defined as any breast milk received in the previous week). Modes of birth included planned cesarean birth, emergency cesarean birth, and spontaneous vaginal birth and induced vaginal birth. Instrument vaginal birth was not considered. Women who planned a cesarean birth were less likely to plan to breastfeed, and women who had vaginal births were significantly more likely to breastfeed at both four weeks (78\% compared to $\sim 70 \%$ in all other groups) and six months (57\% compared to $\sim 47 \%$ in women with planned cesarean and induced vaginal births, and $39 \%$ in those with emergency cesarean births). Only $80 \%$ of women planning a cesarean birth initiated breastfeeding, compared to approximately $85 \%$ in the emergency cesarean and induced vaginal birth groups. Median breastfeeding duration for the different birth modes were 38.7 weeks for planned cesarean birth, 20.6 weeks for emergency cesarean birth, 45 weeks for spontaneous vaginal birth, and 25.8 weeks for induced vaginal birth. While these findings clearly indicate greater breastfeeding success in spontaneous vaginal birth, there was a large difference in sample sizes between the groups. both vaginal birth groups had over 1,000 women, the planned cesarean group had 489 , and the emergency cesarean group had 363. Further, social desirability of breastfeeding may contribute to a reporting bias in these participants.

Of additional breastfeeding relevance, the Term Breech Trial, described below [18] found no difference between planned cesarean and planned vaginal births (with no further differentiation for MOB) at three months postpartum. However fewer women in the cesarean birth group breastfed their infants within the first few hours of life.

\section{Mode of birth and newborn sleep}

Freudigman and Thoman [19] evaluated 51 full-term infants throughout their hospital stay for sleep/wake states and MOB. Twenty-six infants were born vaginally, 12 by emergency cesarean, and 13 by elective cesarean. This allowed for threegroup comparisons for the first two days and for two-group comparison (both cesarean birth types) for the later days. All infants were healthy, although 12 of the original 63 recruited infants were excluded because they spent less than $30 \%$ of the first day of life in their crib. Emergency cesareans were all done due to labor that "failed to progress." Twelve of the 13 elective cesarean births were due to previous cesarean birth, and one was due to suspected macrosomia. Groups were similar on demographic variables with the exception of exclusive breastfeeding; $61.5 \%$ of vaginally born infants, and $33.3 \%$ and $30.8 \%$ of cesarean born infants (emergency and elective, respectively) were exclusively breastfed. Feeding method had no significant effect on infant sleep in this small sample. Baby sleep data were gathered using a well-validated tool involving a mattress pad that recorded infant respirations and body movement whenever the infant was in the crib. Babies were placed in their cribs upon transfer to the newborn nursery, anywhere from 30 to 120 minutes of age, at which time data collection began. Recorders ran for 24 hours, though only in-crib times were used for analyses. This potential bias assumes all infants sleep optimally in the crib. Many infants fall sleep being held or fed, and sleep may be disrupted upon transfer, or may simply sleep more soundly with maternal contact. Despite the small sample, babies born by cesarean had significantly more active sleep (compared to quiet sleep), more sleep-wake transitions, and more waking. They were also significantly lacking in diurnal rhythms, demonstrating "daynight confusion." Of note women underwent elective cesarean birth under general anesthesia in this hospital in 1998, while regional anesthesia is used for the majority of cesarean births today. The authors discuss the possibility of hormonal cues and medications in birth as likely culprits for sleep differences in babies born vaginally compared to abdominally.

The effect of birth method on newborn sleep was assessed in another study involving infants of diabetic mothers [20]. Using actigraphy to record sleep patterns in a sample of 102 infants born to mothers with gestational diabetes and 118 control infants (77\% vaginal and $23 \%$ cesarean), the cesarean born infants spend significantly more time in active sleep than infants born vaginally. This sample was largely Caucasian, and all were born 
at one hospital in Rhode Island. The large sampling of infants of diabetic mothers may limit generalizability of findings to all infants, as the incidence of gestational diabetes is only $2-3 \%$ in the general population.

\section{Summary}

According to allostatic load theory, there are many confounding variables and comorbidities that can play an active role in how physically and emotional stressful MOB can be for both mother and infant. As a result it is difficult to tease out the unique contribution of MOB on newborn outcomes. Regardless, delayed onset of lactation does appear to occur as a result of cesarean birth and subsequent maternal-infant separation. With current shifts in practice, including skin-to-skin care in the operative suite, newer research may see this delay minimized, and therefore the effect minimized. However, MOB remains a clinically significant variable for onset of breastfeeding. Unfortunately, there is little literature exploring the duration of breastfeeding as a result of birth mode, with only one study finding shorter duration of breastfeeding at six months follow up for women who experienced a cesarean birth.

No differences were found in newborn temperament in relation to MOB. Most outcomes need more exploration with larger sample sizes and confounding variables controlled in the analyses. There is preliminary evidence that newborn sleep patterns may be influenced by MOB, and more research is required for this understudied phenomenon. Overall, of all the potential salient outcomes examined in this critical review of literature, MOB appears to have the greatest effect on breastfeeding, specifically initiation and likely duration as well. Therefore, within the framework of decreasing the allostatic load on the infant to optimize adaptation to extra uterine life, stressors in birth should be minimized.

\section{References}

1. Aziz K, Chadwick M, Baker M, Andrews W. Ante- and intrapartum factors that predict increased need for neonatal resuscitation. Resuscitation. 2008;79(3):444-452. doi:10.1016/j resuscitation.2008.08.004.

2. Rajani AK, Chitkara R, Halamek LP. Delivery room management of the newborn. Pediatric Clinics North America. 2009;56(3):515-535. doi:10.1016/j.pcl.2009.03.003.

3. Martin JA, Hamilton BE, Osterman MJK. Births in the United States. 2015. NCHS Data Brief. 2016;258:1-7.

4. Declercq E, Menacker F, Macdorman M. Maternal risk profiles and the primary cesarean rate in the United States, 1991-2002. American Journal of Public Health. 2006;96(5):867-872. doi: 10.2105/ AJPH.2004.052381.

5. Anderson GC. Risk in mother-infant separation postbirth. IMAGE: Journal of Nursing Scholarship. 1989;21(4):196-199.
6. Mercer JS, Erickson-Owens DA, Graves B, Haley MM. Evidencebased practices for the fetal to newborn transition. Journal of Midwifery \& Women's Health. 2007;52(2):262-272. doi:10.1016/j. jmwh.2007.01.005.

7. Yeomans ER. Operative vaginal delivery. Obstetrics and Gynecology. 2010;115(3):645-653. doi:10.1097/AOG.0b013e3181cfbefd.

8. Lupien S, McEwen BS, Gunnar MR, Heim C. Effects of stress throughout the lifespan on the brain, behaviour and cognition. Neuroscience. 2009;10:434-445. doi: 10.1038/nrn2639.

9. BoldtT, Luukkainen P, Fyhrquist F, Pohjavuori M, Andersson S Birthstress increases adrenomedullin in the newborn. ActaPaediatrica. 1998;87(1):93-94. doi: 10.1111/j.1651-2227.1998.tb01393.x.

10. Anand KJS, Scalzo FM. Can adverse neonatal experiences alter brain development and subsequent behavior? Biology of the Neonate. 2000;77(2):69-82. doi:14197.

11. McEwen BS, Stellar E. Stress and the individual, mechanisms leading to disease. Archives of Internal Medicine. 1993;153:2093-2101.

12. Nelson NM, Enkin MW, Saigal S, Bennett KJ, Milner R, Sackett DL. A randomized clinical trial of the Leboyer approach to childbirth. New England Journal of Medicine. 1980;302(12):655-660. DOI:10.1056/ NEJM198003203021203.

13. Maziade $M$, Boudreault $M$, Cote $R$, Thivierge J. Influence of gentle birthdelivery procedures and other perinatal circumstances on infant temperament: Developmental and social implications. The Journal of Pediatrics. 1986;108(1):134-136.

14. Dubignon J, Campbell D, Curtis M, Partington MW. The relation between laboratory measures of sucking, food intake, and perinatal factors during the new born period. Child Development. 1969;40(4):11071120.

15. Chapman DJ, Perez-Escamilla R. Identification of risk factors for delayed onset of lactation. Journal of the American Dietetic Association. 1999;99:450-454. DOI:10.1016/S0002-8223(99)00109-1.

16. Dewey KG, Nommsen-Rivers LA, Heining MJ, Cohen RJ. Risk Factors for Suboptimal Infant Breastfeeding Behavior, Delayed Onset of Lactation, and Excess Neonatal Weight Loss. Pediatrics. 2003;112(3):607-619.

17. Ahluwalia IB, Li R, Morrow B. Breastfeeding practices: Does method of delivery matter? Maternal Child Health Journal. 2012;16:S231-S237. Doi: 10.1007/s10995-012-1093-9.

18. Hannah ME, Hannah WJ, Hodnet ED, Chalmers B, Kung R, Willan A, et al. Outcomes at 3 months after planned cesarean vs planned vaginal delivery for breech presentation at term: The International Randomized Term Breech Trial. Journal of the American Medical Association. 2002;287(14):1822-1831.

19. Freudigman KA, Thoman EB. Infant sleep during the first postnatal day: An opportunity for assessment of vulnerability. Pediatrics. 1993;92(3):373-379.

20.Sadeh A, Dark I, Vohr BR. Newborns' sleep-wake patterns: the role of maternal, delivery and infant factors. Early Human Development.1996;44(2):113-126. 